\title{
Well-balanced high-order centred schemes for non-conservative hyperbolic systems. Applications to shallow water equations with fixed and mobile bed
}

\author{
Alberto Canestrelli ${ }^{\mathrm{a}, *}$, Annunziato Siviglia $^{\mathrm{b}}$, Michael Dumbser $^{\mathrm{b}}$, Eleuterio F. Toro ${ }^{\mathrm{b}}$ \\ a Department IMAGE, University of Padova, Via Loredan 20, I-35131 Padova, Italy \\ ${ }^{\mathrm{b}}$ Department of Civil and Environmental Engineering, University of Trento, Via Mesiano 77, I-38100 Trento, Italy
}

\section{A R T I C L E I N F O}

\section{Article history:}

Received 23 October 2008

Received in revised form 4 February 2009

Accepted 8 February 2009

Available online 26 February 2009

\section{Keywords:}

Non-conservative hyperbolic systems

Centred schemes

High-order WENO finite volume methods

Shallow water equations

Sediment transport

ADER

FORCE

\begin{abstract}
A B S T R A C T
This paper concerns the development of high-order accurate centred schemes for the numerical solution of one-dimensional hyperbolic systems containing non-conservative products and source terms. Combining the PRICE-T method developed in [Toro E, Siviglia A. PRICE: primitive centred schemes for hyperbolic system of equations. Int J Numer Methods Fluids 2003;42:1263-91] with the theoretical insights gained by the recently developed path-conservative schemes [Castro M, Gallardo J, Parés C. High-order finite volume schemes based on reconstruction of states for solving hyperbolic systems with nonconservative products applications to shallow-water systems. Math Comput 2006;75:1103-34; Parés C. Numerical methods for nonconservative hyperbolic systems: a theoretical framework. SIAM J Numer Anal 2006;44:300-21], we propose the new PRICE-C scheme that automatically reduces to a modified conservative FORCE scheme if the underlying PDE system is a conservation law. The resulting first-order accurate centred method is then extended to high order of accuracy in space and time via the ADER approach together with a WENO reconstruction technique. The well-balanced properties of the PRICE-C method are investigated for the shallow water equations. Finally, we apply the new scheme to the shallow water equations with fix bottom topography and with variable bottom solving an additional sediment transport equation.
\end{abstract}

(c) 2009 Elsevier Ltd. All rights reserved.

\section{Centred schemes for non-conservative hyperbolic systems}

We consider systems of hyperbolic partial differential equations of the form

$$
\frac{\partial \mathbf{Q}}{\partial t}+\mathbf{A}(\mathbf{Q}) \frac{\partial \mathbf{Q}}{\partial x}=\mathbf{0}, \quad(x, t) \in \mathbb{R} \times \mathbb{R}_{0}^{+}, \quad \mathbf{Q} \in \Omega \subseteq \mathbb{R}^{N},
$$

in which $\mathbf{Q}=\left[q_{1}, \ldots, q_{N}\right]^{T}$ is the vector of unknowns and $\mathbf{A}=\mathbf{A}(\mathbf{Q})$ is the coefficient matrix. We suppose that the unknown function $\mathbf{Q}=\mathbf{Q}(x, t)$ takes its values inside an open convex set $\Omega$ included in $\mathbb{R}^{N}$ and that $\mathbf{Q} \rightarrow \mathbf{A}(\mathbf{Q})$ is a smooth locally bounded map. We assume system (1) to be hyperbolic with real eigenvalues $\lambda_{1}, \lambda_{2}, \ldots, \lambda_{N}$ and with a full set of corresponding linearly independent right eigenvectors $\mathbf{r}_{1}, \mathbf{r}_{2}, \ldots, \mathbf{r}_{N}$. The numerical methods developed in this paper are of the centred type and will only require an estimate for the maximum signal speed in absolute value in order to satisfy the Courant stability condition for the time step. The vector of unknowns $\mathbf{Q}$ in (1) will be always chosen to be the vector of physically conserved variables. So in the case that $\mathbf{A}(\mathbf{Q})$ is the Jacobian matrix

\footnotetext{
* Corresponding author.

E-mail addresses: canestrelli@idra.unipd.it, albcanes@yahoo.it (A. Canestrelli), nunzio.siviglia@ing.unitn.it (A. Siviglia), michael.dumbser@ing.unitn.it (M. Dumbser), toroe@ing.unitn.it (E.F. Toro).
}

$\mathbf{A}(\mathbf{Q})=\partial \mathbf{F} / \partial \mathbf{Q}$ of some flux function $\mathbf{F}=\mathbf{F}(\mathbf{Q})$, the non-conservative system (1) can be expressed in conservative form

$\frac{\partial \mathbf{Q}}{\partial t}+\frac{\partial \mathbf{F}(\mathbf{Q})}{\partial x}=0$.

In [29] a series of primitive centred (PRICE) numerical schemes for solving systems of hyperbolic partial differential equations written in the non-conservative form (1) have been developed. The most promising of these schemes, namely the PRICE-T scheme, will be the basis of the high-order centred schemes proposed in this article.

\subsection{The FORCE scheme for conservative systems}

Since the PRICE-T scheme [29] is the non-conservative analogue of the conservative FORCE scheme [31,32], that is in turn a deterministic re-interpretation of the staggered-grid version of the Random Choice Method (RCM) of Glimm [15], we briefly recall here the definition of the FORCE scheme for conservation laws. The FORCE scheme for the conservative system (2) can be written either in a two-step staggered-grid version as

$$
\begin{aligned}
& \mathbf{Q}_{i+\frac{1}{2}}^{n+\frac{1}{2}}=\frac{1}{2}\left(\mathbf{Q}_{i}^{n}+\mathbf{Q}_{i+1}^{n}\right)-\frac{1}{2} \frac{\Delta t}{\Delta x}\left[\mathbf{F}\left(\mathbf{Q}_{i+1}^{n}\right)-\mathbf{F}\left(\mathbf{Q}_{i}^{n}\right)\right], \\
& \mathbf{Q}_{i}^{n+1}=\frac{1}{2}\left(\mathbf{Q}_{i-\frac{1}{2}}^{n+\frac{1}{2}}+\mathbf{Q}_{i+\frac{1}{2}}^{n+\frac{1}{2}}\right)-\frac{1}{2} \frac{\Delta t}{\Delta x}\left[\mathbf{F}\left(\mathbf{Q}_{i+\frac{1}{2}}^{n+\frac{1}{2}}\right)-\mathbf{F}\left(\mathbf{Q}_{i-\frac{1}{2}}^{n+\frac{1}{2}}\right)\right],
\end{aligned}
$$


or in a more convenient conservative non-staggered one-step formulation with two-point fluxes as

$\mathbf{Q}_{i}^{n+1}=\mathbf{Q}_{i}^{n}-\frac{\Delta t}{\Delta x}\left[\mathbf{F}_{i+\frac{1}{2}}^{\mathrm{FORCE}}-\mathbf{F}_{i-\frac{1}{2}}^{\mathrm{FORC}}\right]$.

Here, the FORCE flux $\mathbf{F}_{i+\frac{1}{2}}^{\mathrm{FOR}}$ is the arithmetic average of the LaxFriedrichs and the Lax-Wendroff flux, i.e.

$\mathbf{F}_{i+\frac{1}{2}}^{\mathrm{FORCE}}=\frac{1}{2}\left(\mathbf{F}_{i+\frac{1}{2}}^{\mathrm{LF}}+\mathbf{F}_{i+\frac{1}{2}}^{\mathrm{LW}}\right)$,

with the Lax-Friedrichs flux

$\mathbf{F}_{i+\frac{1}{2}}^{\mathrm{LF}}=\frac{1}{2}\left[\mathbf{F}\left(\mathbf{Q}_{i+1}^{n}\right)+\mathbf{F}\left(\mathbf{Q}_{i}^{n}\right)\right]-\frac{1}{2} \frac{\Delta x}{\Delta t}\left(\mathbf{Q}_{i+1}^{n}-\mathbf{Q}_{i}^{n}\right)$

and the Lax-Wendroff flux

$\mathbf{F}_{i+\frac{1}{2}}^{\mathrm{LW}}=\mathbf{F}\left(\mathbf{Q}_{i+\frac{1}{2}}^{n+\frac{1}{2}}\right)$,

where $\mathbf{Q}_{i+\frac{1}{2}}^{n+\frac{1}{2}}$ is given by (3). It is easy to prove via simple algebraic manipulations that the two schemes (3) \& (4) and (5)-(8) are identical. For the purpose of this paper the Lax-Wendroff scheme as given in (8)-(3), is not convenient. The main problem is its two-step nature and the resulting non-linearity of the numerical flux function with respect to the arguments $\mathbf{Q}_{i}^{n}, \mathbf{Q}_{i+1}^{n}, \mathbf{F}\left(\mathbf{Q}_{i}^{n}\right)$ and $\mathbf{F}\left(\mathbf{Q}_{i+1}^{n}\right)$, which makes it cumbersome for further analytic manipulations, since we do not want to make any further assumptions on $\mathbf{F}$, other than hyperbolicity. We therefore propose the following variant of the conservative FORCE flux:

$\mathbf{F}_{i+\frac{1}{2}}^{\mathrm{FORCE}^{\prime}}=\frac{1}{2}\left(\mathbf{F}_{i+\frac{1}{2}}^{\mathrm{LF}}+\mathbf{F}_{i+\frac{1}{2}}^{\mathrm{LW}^{\prime}}\right)$

where the modified Lax-Wendroff-type flux is now given by

$\mathbf{F}_{i+\frac{1}{2}}^{\mathrm{LW}}=\frac{1}{2}\left[\mathbf{F}\left(\mathbf{Q}_{i+1}^{n}\right)+\mathbf{F}\left(\mathbf{Q}_{i}^{n}\right)\right]-\frac{1}{2} \frac{\Delta t}{\Delta x} \widehat{\mathbf{A}}_{i+\frac{1}{2}}\left[\mathbf{F}\left(\mathbf{Q}_{i+1}^{n}\right)-\mathbf{F}\left(\mathbf{Q}_{i}^{n}\right)\right]$.

The matrix $\widehat{\mathbf{A}}_{i+\frac{1}{2}}=\widehat{\mathbf{A}}_{i+\frac{1}{2}}\left(\mathbf{Q}_{i}^{n}, \mathbf{Q}_{i+1}^{n}\right)$ is a function of the left and the right states and still it has to be chosen appropriately. For linear systems with constant coefficient matrix A, the fluxes given by (8) \& (3) and (10) are identical. We point out that the modified LaxWendroff-type flux (10) has to be introduced for technical reasons, in order to be able to prove later on that the proposed non-conservative centred schemes reduce exactly to the conservative centred scheme (5) with the modified FORCE flux (9), if the matrix $\mathbf{A}(\mathbf{Q})$ is the Jacobian of some flux function $\mathbf{F}(\mathbf{Q})$.

\subsection{The original two-step PRICE-T scheme}

The PRICE-T scheme introduced in [29] for non-conservative systems of the form (1) is the following two-step scheme:

$\mathbf{Q}_{i+\frac{1}{2}}^{n+\frac{1}{2}}=\frac{1}{2}\left(\mathbf{Q}_{i}^{n}+\mathbf{Q}_{i+1}^{n}\right)-\frac{1}{2} \frac{\Delta t}{\Delta x} \widehat{\mathbf{A}}_{i+\frac{1}{2}}\left(\mathbf{Q}_{i+1}^{n}-\mathbf{Q}_{i}^{n}\right)$

$\mathbf{Q}_{i}^{n+1}=\frac{1}{2}\left(\mathbf{Q}_{i-\frac{1}{2}}^{n+\frac{1}{2}}+\mathbf{Q}_{i+\frac{1}{2}}^{n+\frac{1}{2}}\right)-\frac{1}{2} \frac{\Delta t}{\Delta x} \widehat{\mathbf{A}}_{i}\left(\mathbf{Q}_{i+\frac{1}{2}}^{n+\frac{1}{2}}-\mathbf{Q}_{i-\frac{1}{2}}^{n+\frac{1}{2}}\right)$,

where the matrices are evaluated as follows:

$\widehat{\mathbf{A}}_{i}=\mathbf{A}\left(\frac{1}{2}\left[\mathbf{Q}_{i-\frac{1}{2}}^{n+\frac{1}{2}}+\mathbf{Q}_{i+\frac{1}{2}}^{n+\frac{1}{2}}\right]\right), \quad \widehat{\mathbf{A}}_{i+\frac{1}{2}}=\mathbf{A}\left(\frac{1}{2}\left[\mathbf{Q}_{i}^{n}+\mathbf{Q}_{i+1}^{n}\right]\right)$.

When applied to the linear scalar advection equation $q_{t}+\lambda q_{x}=0$, in [29] it was found that the PRICE-T scheme is first-order accurate, monotone and obeys the standard CFL stability condition

$c=\lambda \frac{\Delta t}{\Delta x} \leqslant 1$

where $c$ is the CFL number. It was shown in [29] that for the shallow water equations the scheme (11) and (12) provides a reasonable approximation of weak shock waves, but in the presence of strong shocks the scheme is unable to capture neither the exact position of the front nor the exact post-shock values. In fact, the theorem of Hou and LeFloch [20] states that non-conservative methods will converge to the wrong solution in the presence of shock waves.

It is therefore the declared objective of this contribution to create a modified PRICE-T scheme that automatically reduces to the modified conservative FORCE scheme (5) and (9) in the case $\mathbf{A}(\mathbf{Q})$ is the Jacobian matrix of some flux function $\mathbf{F}(\mathbf{Q})$, i.e. when $\mathbf{A}(\mathbf{Q})=\partial \mathbf{F} / \partial \mathbf{Q}$. The relevance of this result will be noteworthy: it has been proven that in the conservative case that FORCE is the optimal centred scheme resulting from a convex average of (8) and (7) in the sense that it is the least dissipative of all three-point centred methods that are monotone and have stability condition (14), see [32] for details. Also the conservative FORCE scheme has been shown to be convergent in the case of two particular nonlinear hyperbolic systems [8].

Furthermore, we are also looking for a scheme that preserves some particular equilibria of the governing PDE (well-balanced property) and that is easily extendable to high order of accuracy in space and time.

\subsection{The PRICE-R scheme}

We note that the system (1) contains a non-conservative product which, in general, does not make sense in the classical framework of the theory of distributions. With the theory developed by Dal Maso et al. [9], a rigorous definition of weak solutions can be given using a family of paths $\boldsymbol{\Psi}=\boldsymbol{\Psi}\left(\mathbf{Q}_{L}, \mathbf{Q}_{R}, s\right)$ connecting two states $\mathbf{Q}_{L}$ and $\mathbf{Q}_{R}$ across a discontinuity with $(s \in[0,1])$. For all numerical test cases presented in this paper, we always use the simple segment path, given by

$\boldsymbol{\Psi}\left(\mathbf{Q}_{L}, \mathbf{Q}_{R}, s\right)=\mathbf{Q}_{L}+s\left(\mathbf{Q}_{R}-\mathbf{Q}_{L}\right)$

Once a family of paths is chosen, it is possible to give a sense to the non-conservative product at discontinuities as a Borel measure (see [9] for details). Moreover, based on the theoretical advances in [9], generalizations of the Roe method to systems of the form (1) have been introduced in $[33,5,26]$. Given a family of paths $\boldsymbol{\Psi}$, a matrix $\mathbf{A}_{\boldsymbol{\Psi}}$ is called a Roe matrix if it satisfies the following properties:

- for any $\mathbf{Q}_{L}, \mathbf{Q}_{R} \in \Omega, \mathbf{A}_{\boldsymbol{\Psi}}\left(\mathbf{Q}_{L}, \mathbf{Q}_{R}\right)$ has $N$ real eigenvalues;

- $\mathbf{A}_{\Psi}(\mathbf{Q}, \mathbf{Q})=\mathbf{A}(\mathbf{Q})$, for any $\mathbf{Q} \in \Omega$;

- for any $\mathbf{Q}_{L}, \mathbf{Q}_{R} \in \Omega$ :

$\mathbf{A}_{\boldsymbol{\Psi}}\left(\mathbf{Q}_{\mathrm{L}}, \mathbf{Q}_{R}\right)\left(\mathbf{Q}_{R}-\mathbf{Q}_{\mathrm{L}}\right)=\int_{0}^{1} \mathbf{A}\left(\boldsymbol{\Psi}\left(s, \mathbf{Q}_{\mathrm{L}}, \mathbf{Q}_{R}\right)\right) \frac{\partial \boldsymbol{\Psi}}{\partial s} d s$.

In the case when $\mathbf{A}(\mathbf{Q})$ is the Jacobian matrix of a flux $\mathbf{F}(\mathbf{Q})$, then (16) is independent of the choice of the path and we have the classical Roe property:

$\mathbf{A}_{\boldsymbol{\Psi}}\left(\mathbf{Q}_{L}, \mathbf{Q}_{R}\right)\left(\mathbf{Q}_{R}-\mathbf{Q}_{L}\right)=\mathbf{F}\left(\mathbf{Q}_{R}\right)-\mathbf{F}\left(\mathbf{Q}_{L}\right)$.

With this insight, we now consider a modified version of the PRICE$T$ scheme, called PRICE-R in the following, where we evaluate the matrices $\mathbf{A}_{i}$ and $\mathbf{A}_{i+\frac{1}{2}}$ in Eqs. (11) and (12) as

$\widehat{\mathbf{A}}_{i}=\mathbf{A}_{\boldsymbol{\Psi}}\left(\mathbf{Q}_{i-\frac{1}{2}}^{n+\frac{1}{2}}, \mathbf{Q}_{i+\frac{1}{2}}^{n+\frac{1}{2}}\right), \quad \widehat{\mathbf{A}}_{i+\frac{1}{2}}=\mathbf{A}_{\Psi}\left(\mathbf{Q}_{i}^{n}, \mathbf{Q}_{i+1}^{n}\right)$

Using algebraic manipulations and Eq. (17), it is easy to prove that the scheme (11) \& (12) with (18) reduces to the original conservative FORCE scheme (3) \& (4) and therefore to (5) with the original FORCE flux (6) with (7) and (8), if $\mathbf{A}(\mathbf{Q})$ is the Jacobian matrix of a flux $\mathbf{F}(\mathbf{Q})$.

The choice of the matrices given by (18) has the advantage that the resulting PRICE-R method becomes exactly conservative if applied to conservation laws. However, it has the obvious 
disadvantage that one needs to compute the Roe matrix, which may become very cumbersome or even impossible for complicated hyperbolic systems. Since we are interested in a truly centred approach that does not need any wave propagation information contained in the underlying governing PDE, we therefore do not want to compute the Roe matrix explicitly. An obvious alternative to the analytical computation of the Roe-type matrix $\mathbf{A}_{\boldsymbol{\Psi}}$ is to use definition (16) and the segment path (15), which yields

$\mathbf{A}_{\boldsymbol{\Psi}}\left(\mathbf{Q}_{L}, \mathbf{Q}_{R}\right)\left(\mathbf{Q}_{R}-\mathbf{Q}_{L}\right)=\left(\int_{0}^{1} \mathbf{A}\left(\boldsymbol{\Psi}\left(s, \mathbf{Q}_{L}, \mathbf{Q}_{R}\right)\right) d s\right)\left(\mathbf{Q}_{R}-\mathbf{Q}_{L}\right)$

Hence, we obtain the following definition of the Roe matrix $\mathbf{A}_{\boldsymbol{\Psi}}$ in the case of a segment path:

$\mathbf{A}_{\boldsymbol{\Psi}}\left(\mathbf{Q}_{L}, \mathbf{Q}_{R}\right)=\int_{0}^{1} \mathbf{A}\left(\boldsymbol{\Psi}\left(s, \mathbf{Q}_{L}, \mathbf{Q}_{R}\right)\right) d s$.

It is now a key idea of this article to compute the Roe matrix $\mathbf{A}_{\boldsymbol{\Psi}} \mathrm{di}$ rectly using the integral along the segment path $\Psi$, as given by the right-hand side of Eq. (20). The exact conservation properties of the PRICE-R schemes described above are still valid in this case, if the integral is computed exactly. For complicated nonlinear hyperbolic systems, the exact computation of the integral may quickly become too cumbersome, so that we propose to resort to classical high-order accurate Gaussian quadrature rules to compute the right-hand side of Eq. (20) numerically. Given an $M$-point Gaussian quadrature rule with weights $\omega_{j}$ and positions $s_{j}$ distributed in the unit interval $[0 ; 1]$, a very accurate numerical approximation of the Roe matrix $\mathbf{A}_{\boldsymbol{\Psi}}$ is given by the following centred Roe-type matrix:

$\mathbf{A}_{\boldsymbol{\Psi}}^{M}\left(\mathbf{Q}_{L}, \mathbf{Q}_{R}\right)=\sum_{j=1}^{M} \omega_{j} \mathbf{A}\left(\boldsymbol{\Psi}\left(s_{j}, \mathbf{Q}_{L}, \mathbf{Q}_{R}\right)\right)$

Recall that an $M$-point Gaussian quadrature rule integrates polynomials up to degree $2 M-1$ exactly, which means that one Gaussian point is enough if the system matrix $\mathbf{A}(\mathbf{Q})$ is a linear function in $\mathbf{Q}$. In order to study the sensitivity of the resulting PRICE-R scheme using the approximate Roe matrix (21) we show the behaviour of the method for the shallow water equations in the presence of a strong shock wave. The results are depicted in the top row of Fig. 1. The computations are carried out with different numbers of Gaussian points. It appears as if with three or more Gaussian points the solution cannot be distinguished any more from the solution obtained using the exact Roe matrix. In the bottom row we show the behaviour of the scheme using three Gaussian points and different numbers of cells. We highlight that in this way conservation is not maintained exactly any more but it can be preserved numerically up to any desired order of accuracy by simply increasing the number of Gaussian points. For even more sophisticated methods in computing the path integral numerically, one even could think of using adaptive and extrapolation strategies, such as Romberg integration. However, for all the test cases presented in this article, three Gaussian quadrature points have shown to be enough. We also note that the dam-break test considered in Fig. 1 is not physical at all but is just used to show the quality and the robustness of the numerical method since the right initial velocity of $-3000 \mathrm{~m} / \mathrm{s}$ and the left initial depth of $1000 \mathrm{~m}$ are never reached in real situations. So for any application of shallow water equations the numerical approximation given by Eq. (21) can be considered a good choice. Finally the reader can easily verify that the original PRICE-T scheme (11) and (12) with the matrices $\widehat{\mathbf{A}}_{i}$ and $\widehat{\mathbf{A}}_{i+\frac{1}{2}}$ given by (13) can be reinterpreted as the PRICE-R scheme, where Eq. (21) is approximated with just one single Gaussian point. This choice was shown in [29] already to give reasonable shock-capturing properties in the case of weak shocks. Note that the centred Roe-type matrix $\mathbf{A}_{\Psi}^{M}$ could also be used in the class of centred schemes developed in [7].

We would finally like to remark that even exactly path-conservative schemes may fail to converge for non-conservative systems as reported recently in [6].
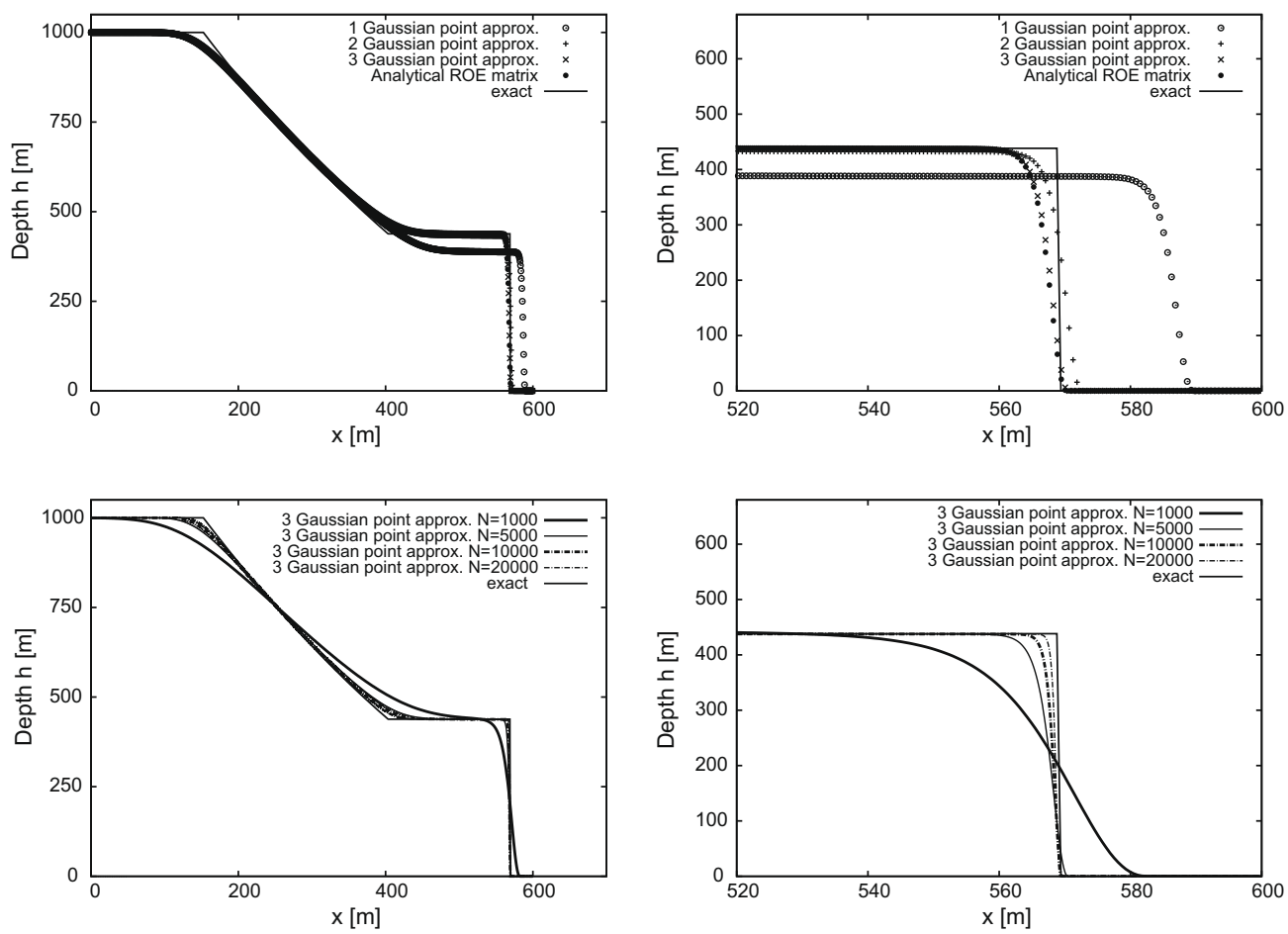

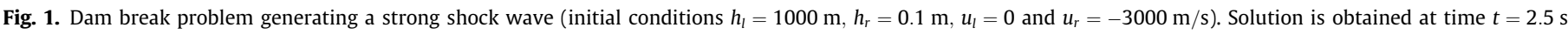

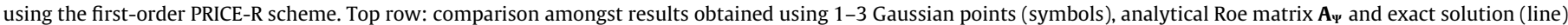

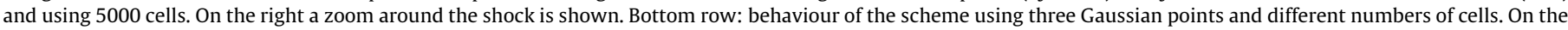
right a zoom around the shock is shown. 


\subsection{Alternative formulation of the PRICE-R scheme}

After some algebra, the two-step PRICE-T scheme given by (11) and (12) can be rewritten as a one-step scheme, as follows:

$\mathbf{Q}_{i}^{n+1}=\mathbf{Q}_{i}^{n}-\frac{\Delta t}{\Delta x}\left[\mathbf{A}_{i+\frac{1}{2}}^{-}\left(\mathbf{Q}_{i+1}^{n}-\mathbf{Q}_{i}^{n}\right)+\mathbf{A}_{i-\frac{1}{2}}^{+}\left(\mathbf{Q}_{i}^{n}-\mathbf{Q}_{i-1}^{n}\right)\right]$,

where

$\widehat{\mathbf{A}}_{i+\frac{1}{2}}^{-}=\frac{1}{4}\left[\widehat{\mathbf{A}}_{i}-\frac{\Delta x}{\Delta t} \mathbf{I}+\widehat{\mathbf{A}}_{i+\frac{1}{2}}-\frac{\Delta t}{\Delta x} \widehat{\mathbf{A}}_{i} \widehat{\mathbf{A}}_{i+\frac{1}{2}}\right]$

and

$\widehat{\mathbf{A}}_{i-\frac{1}{2}}^{+}=\frac{1}{4}\left[\widehat{\mathbf{A}}_{i}+\frac{\Delta x}{\Delta t} \mathbf{I}+\widehat{\mathbf{A}}_{i-\frac{1}{2}}+\frac{\Delta t}{\Delta x} \widehat{\mathbf{A}}_{i} \widehat{\mathbf{A}}_{i-\frac{1}{2}}\right]$,

with the identity matrix I and all matrices $\widehat{\mathbf{A}}$ computed as in (18). We emphasize on the identical form of this scheme with the path-conservative Roe scheme proposed in [26,5,4], the only difference being in the matrices $\mathbf{A}_{i+\frac{1}{2}}^{-}$and $\mathbf{A}_{i-\frac{1}{2}}^{+}$. In our case, these Roe-type matrices are centred, that is they do not use explicit wave properties information. Moreover, they are computed numerically, whereas in $[5,4]$ they are computed as

$\mathbf{A}_{i+\frac{1}{2}}^{ \pm}=\mathbf{A}_{\boldsymbol{\Psi}}\left(\mathbf{Q}_{i}^{n}, \mathbf{Q}_{i+1}^{n}\right)^{ \pm}=\mathbf{R}_{\boldsymbol{\Psi}} \mathbf{\Lambda}_{\Psi}^{ \pm} \mathbf{R}_{\boldsymbol{\Psi}}^{-1}$.

Here, the usual definitions are applied, i.e. $\mathbf{R}_{\Psi}$ is the matrix of right eigenvectors of the Roe matrix $\mathbf{A}_{\Psi}$ and $\boldsymbol{\Lambda}_{\boldsymbol{\Psi}}$ is the diagonal matrix with the eigenvalues of $\mathbf{A}_{\boldsymbol{\Psi}}$. The matrices $\boldsymbol{\Lambda}_{\boldsymbol{\Psi}}^{ \pm}$are, as usual, either the positive or the negative part of the diagonal matrix $\Lambda_{\Psi}$. For very complicated non-conservative systems one could still construct an upwind method by using $\mathbf{A}_{i+\frac{1}{2}}^{ \pm}=\mathbf{A}_{\Psi}^{M}\left(\mathbf{Q}_{i}^{n}, \mathbf{Q}_{i+1}^{n}\right)^{ \pm}$and computing the eigenstructure fully numerically, e.g. using the RG subroutine of the EISPACK library. We emphasize that the use of $\mathbf{A}_{\boldsymbol{\psi}}^{M}$ instead of $\mathbf{A}_{\Psi}$ in (25) still has the advantage that the Roe-averages do not have to be computed analytically, which may be very difficult or even impossible for very general nonlinear systems, however the necessary numerical computation of the full eigenstructure is very costly.

So the basic idea of our new PRICE-C scheme presented in the following section is to avoid the use of the analytical Roe matrix and the computation of $\mathbf{A}_{i+\frac{1}{2}}^{ \pm}$, which requires the knowledge of wave propagation information (upwind philosophy), and to use instead only the centred Roe-type matrix $\mathbf{A}_{\Psi}^{M}$ which is computed numerically with a number of Gaussian points that is adequate for the problem to be solved (centred philosophy).

\subsection{The PRICE-C scheme}

The main drawback of the scheme (22)-(24) is that the matrices $\mathbf{A}_{i-\frac{1}{2}}^{+}$and $\mathbf{A}_{i+\frac{1}{2}}^{-}$are three-point functions, i.e. each of them depends on the three states $\mathbf{Q}_{i-1}^{n}, \mathbf{Q}_{i}^{n}$ and $\mathbf{Q}_{i+1}^{n}$. This prevents a direct extension of the PRICE-R method to multiple space dimensions and high order of accuracy using a polynomial reconstruction of $\mathbf{Q}$.

To circumvent this problem, we therefore propose to modify the matrices $\mathbf{A}_{i-\frac{1}{2}}^{+}$and $\mathbf{A}_{i+\frac{1}{2}}^{-}$, substituting the matrix $\widehat{\mathbf{A}}_{i}$ in (23) with $\widehat{\mathbf{A}}_{i+\frac{1}{2}}$ and the matrix $\widehat{\mathbf{A}}_{i}$ in (24) with $\widehat{\mathbf{A}}_{i-\frac{1}{2}}$, in order to make them only two-point functions of the two adjacent states. After these modifications, the final non-conservative version of the FORCE method, called PRICE-C scheme in the following, reads as follows:

$\mathbf{Q}_{i}^{n+1}=\mathbf{Q}_{i}^{n}-\frac{\Delta t}{\Delta x}\left[\mathbf{A}_{i+\frac{1}{2}}^{-}\left(\mathbf{Q}_{i+1}^{n}-\mathbf{Q}_{i}^{n}\right)+\mathbf{A}_{i-\frac{1}{2}}^{+}\left(\mathbf{Q}_{i}^{n}-\mathbf{Q}_{i-1}^{n}\right)\right]$,

with

$\mathbf{A}_{i+\frac{1}{2}}^{-}=\frac{1}{4}\left[2 \mathbf{A}_{\Psi}^{M}\left(\mathbf{Q}_{i}^{n}, \mathbf{Q}_{i+1}^{n}\right)-\frac{\Delta x}{\Delta t} \mathbf{I}-\frac{\Delta t}{\Delta x}\left(\mathbf{A}_{\Psi}^{M}\left(\mathbf{Q}_{i}^{n}, \mathbf{Q}_{i+1}^{n}\right)\right)^{2}\right]$ and

$\mathbf{A}_{i-\frac{1}{2}}^{+}=\frac{1}{4}\left[2 \mathbf{A}_{\Psi}^{M}\left(\mathbf{Q}_{i-1}^{n}, \mathbf{Q}_{i}^{n}\right)+\frac{\Delta x}{\Delta t} \mathbf{I}+\frac{\Delta t}{\Delta x}\left(\mathbf{A}_{\Psi}^{M}\left(\mathbf{Q}_{i-1}^{n}, \mathbf{Q}_{i}^{n}\right)\right)^{2}\right]$

Now the matrices $\mathbf{A}_{i+\frac{1}{2}}^{-}$and $\mathbf{A}_{i-\frac{1}{2}}^{+}$only depend on two adjacent states. With the properties (20) and (17) it can be easily proven that if the $\operatorname{PDE}(1)$ is a conservation law (2), then we have

$\mathbf{A}_{i+\frac{1}{2}}^{-}\left(\mathbf{Q}_{i+1}^{n}-\mathbf{Q}_{i}^{n}\right)=\mathbf{F}_{i+\frac{1}{2}}^{\mathrm{FORE}^{\prime}}-\mathbf{F}\left(\mathbf{Q}_{i}^{n}\right)$,

$\mathbf{A}_{i-\frac{1}{2}}^{+}\left(\mathbf{Q}_{i}^{n}-\mathbf{Q}_{i-1}^{n}\right)=\mathbf{F}\left(\mathbf{Q}_{i}^{n}\right)-\mathbf{F}_{i-\frac{1}{2}}^{\mathrm{FORCE}^{\prime}}$

Therefore, the PRICE-C scheme (26)-(28) reduces to the modified conservative FORCE method (5), (9) and (10) if A is the Jacobian of a flux $\mathbf{F}$.

We note that, independently of the present work, a similar method has been proposed in [7], however, with the important difference that in our case the centred Roe-type matrices $\mathbf{A}_{\psi}^{M}$ are computed via an entirely numerical procedure, using $M$-point Gaussian quadrature of appropriate order to evaluate the path integral in (21), whereas in [7] the Roe matrices are computed using analytical expressions for the Roe averages.

We emphasize that our formulation has the important advantage that an explicit computation of the Roe averages is not necessary, following the original philosophy of centred schemes that by definition do not need any additional information on the PDE system. At the same time conservation can be practically maintained up to any desired precision using Gaussian quadrature rules of appropriate order of accuracy. For complicated nonlinear PDE, as they typically arise in industrial, civil and environmental engineering, closed analytical expressions for the Roe averages may be impossible to obtain for a given PDE system. An example for this will be shown later when we consider shallow-water-type systems with moving bed using a complex closure relation.

\section{High-order extension}

\subsection{Nonlinear reconstruction technique}

In this section we briefly discuss the proposed nonlinear weighted essentially non-oscillatory (WENO) reconstruction procedure to reconstruct higher order polynomial data within each spatial cell $T_{i}=\left[x_{i-\frac{1}{2}} ; x_{i+\frac{1}{2}}\right]$ at time $t^{n}$ from the given cell averages $\mathbf{Q}_{i}^{n}$. We emphasize already at this point that the reconstruction procedure is nonlinear and depends strongly on the input data $\mathbf{Q}_{i}^{n}$. Thus, the resulting numerical scheme, even when applied to a completely linear PDE, will be nonlinear and thus it will not be possible to give a closed expression of the scheme.

The reconstruction procedure described here for the onedimensional case follows directly from the guidelines given in [12] for general unstructured two- and three-dimensional meshes. It reconstructs entire polynomials, as the original ENO approach proposed by Harten et al. in [19]. However, we formally write our method like a WENO scheme $[21,23]$ with a particularly simple choice for the linear weights. The most important difference in our approach compared to classical WENO schemes is that standard WENO methods reconstruct point values at the Gaussian integration points instead of an entire polynomial valid inside each element $T_{i}$.

Reconstruction is done for each element on a reconstruction stencil $\mathcal{S}_{i}^{s}$, which is given by the following union of the element $T_{i}$ and its neighbors $T_{j}$,

$\mathcal{S}_{i}^{s}=\bigcup_{j=i+s-k}^{i+s+k} T_{j}$

where $s$ is the stencil shift with respect to the central element $T_{i}$ and $k$ is the spatial extension of the stencil to the left and the right. A 
central reconstruction stencil is given by $s=0$, an entirely left-sided stencil is given by $s=-k$ and an entirely right-sided stencil is given by $s=k$. In our approach, we will always use the three fixed reconstruction stencils $\mathcal{S}_{i}^{0}, \mathcal{S}_{i}^{-k}$ and $\mathcal{S}_{i}^{k}$.

Given the cell average data $\mathbf{Q}_{i}^{n}$ in all elements $T_{i}$ we are looking for a spatial reconstruction polynomial obtained from $\mathcal{S}_{i}^{s}$ at time $t^{n}$ of the form

$\mathbf{w}_{i}^{s}\left(x, t^{n}\right)=\sum_{l=0}^{N} \Psi_{l}(x) \hat{\mathbf{w}}_{l}^{(i, s)}\left(t^{n}\right):=\Psi_{l}(x) \hat{\mathbf{w}}_{l}^{(i, s)}\left(t^{n}\right)$,

where we use the rescaled Legendre polynomials for the spatial reconstruction basis functions $\Psi_{l}(x)$ such that the $\Psi_{l}(x)$ form an orthogonal basis on the element $T_{i}$. In the following, we will use standard tensor index notation, implying summation over indices appearing twice. The number of polynomial coefficients (degrees of freedom) is $L=N+1$, where $N$ is the degree of the reconstruction polynomial. To compute the reconstruction polynomial $\mathbf{w}_{i}\left(x, t^{n}\right)$ valid for element $T_{i}$ we require integral conservation for all elements $T_{j}$ inside the stencil $\mathcal{S}_{i}^{s}$, i.e.

$\frac{1}{\Delta x} \int_{T_{j}} \mathbf{w}_{i}^{s}\left(x, t^{n}\right) d x=\frac{1}{\Delta x} \int_{T_{j}} \Psi_{l}(x) d x \cdot \hat{\mathbf{w}}_{l}^{(i, s)}\left(t^{n}\right)=\mathbf{Q}_{j}^{n}, \quad \forall T_{j} \in \mathcal{S}_{i}^{s}$.

Eq. (33) yields a linear equation system of the form

$B_{j l} \cdot \hat{\mathbf{w}}_{l}^{(i, s)}\left(t^{n}\right)=\mathbf{Q}_{j}^{n}$

for the unknown coefficients $\hat{\mathbf{w}}_{l}^{(i, s)}\left(t^{n}\right)$ of the reconstruction polynomial on stencil $\mathcal{S}_{i}^{s}$. Since we choose $k=N / 2$ for even $N$ and $k=(N+1) / 2$ for odd $N$, the number of elements in $\mathcal{S}_{i}^{s}$ may become larger than the number of degrees of freedom $L$. In this case, we use a constrained least-squares technique according to [12] to solve (34).

To obtain the final non-oscillatory reconstruction polynomials for each element $T_{i}$ at time $t^{n}$, we finally construct a datadependent nonlinear combination of the polynomials $\mathbf{w}_{i}^{0}\left(x, t^{n}\right)$, $\mathbf{w}_{i}^{-k}\left(x, t^{n}\right)$ and $\mathbf{w}_{i}^{k}\left(x, t^{n}\right)$ obtained from the central, left-sided and right-sided stencils as follows:

$\mathbf{w}_{i}\left(x, t^{n}\right)=\hat{\mathbf{w}}_{l}^{i}\left(t^{n}\right) \Psi_{l}(x)$,

with

$\hat{\mathbf{w}}_{l}^{i}\left(t^{n}\right)=\omega_{0} \hat{\mathbf{w}}_{l}^{(i, 0)}\left(t^{n}\right)+\omega_{-k} \hat{\mathbf{w}}_{l}^{(i,-k)}\left(t^{n}\right)+\omega_{k} \hat{\mathbf{w}}_{l}^{(i, k)}\left(t^{n}\right)$.

The nonlinear weights $\omega_{s}$ are given by the relations

$\omega_{s}=\frac{\tilde{\omega}_{s}}{\tilde{\omega}_{0}+\tilde{\omega}_{-k}+\tilde{\omega}_{k}}, \quad \tilde{\omega}_{s}=\frac{\lambda_{s}}{\left(\sigma_{s}+\epsilon\right)^{r}}$.

In our particular formulation, the oscillation indicators $\sigma_{s}$ are computed from

$\sigma_{s}=\Sigma_{\operatorname{lm}} \hat{\mathbf{w}}_{l}^{s}\left(t^{n}\right) \hat{\mathbf{w}}_{m}^{s}\left(t^{n}\right)$,

with

$\Sigma_{l m}=\sum_{\alpha=1}^{N} \int_{0}^{1} \Delta x^{2 \alpha-1} \frac{\partial^{\alpha} \Psi_{l}(x)}{\partial x^{\alpha}} \cdot \frac{\partial^{\alpha} \Psi_{m}(x)}{\partial x^{\alpha}} d x$.

Here, $\Sigma_{l m}$ is the oscillation indicator matrix for element $T_{i}$. If all computations are done in a reference element, then this matrix does depend neither on the problem nor on the mesh, see [12]. The parameters $\epsilon$ and $r$ are constants for which we typically choose $\epsilon=10^{-14}$ and $r=8$. For the linear weights $\lambda_{s}$ we choose $\lambda_{-k}=\lambda_{k}=1$ and a very large linear weight $\lambda_{0}$ on the central stencil, typically $\lambda_{0}=10^{5}$. It has been shown previously $[21,23]$ that the numerical results are quite insensitive to the WENO parameters $\epsilon$ and $r$ and also with respect to the linear weight on the central stencil $\lambda_{0}$, see [12].

The proposed reconstruction usually uses the accurate and linearly stable central stencil reconstruction in those regions of $\Omega$ where the solution is smooth because of the large linear weight $\lambda_{0}$. However, due to the strongly nonlinear dependence of the weights $\omega_{s}$ on the oscillation indicators $\sigma_{s}$, in the presence of discontinuities the smoother left- or right-sided stencils are preferred, as for standard ENO and WENO methods. For the nonlinear scalar case, the reconstruction operator described above can be directly applied to the cell averages $\mathbf{Q}_{i}^{n}$ of the conserved quantity $\mathbf{Q}$. For nonlinear hyperbolic systems, the reconstruction should be done in characteristic variables $[19,13]$ in order to avoid spurious oscillations that may appear when applying ENO or WENO reconstruction operators component-wise to nonlinear hyperbolic systems.

\subsection{High-order accurate one-step time discretization}

The result of the reconstruction procedure is a non-oscillatory spatial polynomial $\mathbf{w}_{i}\left(x, t^{n}\right)$ defined at time $t^{n}$ inside each spatial element $T_{i}$. However, we still need to compute the temporal evolution of these polynomials inside each space-time element $\left[x_{i-\frac{1}{2}} ; x_{i+\frac{1}{2}}\right] \times\left[t^{n} ; t^{n+1}\right]$ in order to be able to construct our final high-order accurate one-step finite volume scheme. In order to obtain a high-order accurate one-step method we use the ADER approach of Titarev and Toro [30]. The key idea therein is to solve high order Riemann problems at the element boundaries, this is accomplished by a Taylor series expansion in time, use of the Cauchy-Kovalewski procedure and solutions of classical Riemann problems, the state variables and its spatial derivatives. In this paper we adopt the following strategy: We expand the local solution $\mathbf{Q}_{i}(x, t)$ of the PDE in each cell in a space-time Taylor series with respect to the element barycentre $x_{i}$

$$
\begin{aligned}
\mathbf{Q}_{i}(x, t)= & \mathbf{Q}\left(x_{i}, t^{n}\right)+\left(x-x_{i}\right) \frac{\partial \mathbf{Q}}{\partial x}+\left(t-t^{n}\right) \frac{\partial \mathbf{Q}}{\partial t}+\frac{1}{2}\left(x-x_{i}\right)^{2} \frac{\partial^{2} \mathbf{Q}}{\partial x^{2}} \\
& +\left(x-x_{i}\right)\left(t-t^{n}\right) \frac{\partial^{2} \mathbf{Q}}{\partial t \partial x}+\frac{1}{2}\left(t-t^{n}\right)^{2} \frac{\partial^{2} \mathbf{Q}}{\partial t^{2}}+\cdots,
\end{aligned}
$$

where we then use the classical Cauchy-Kovalewski procedure in order to substitute time derivatives with space derivatives, using repeated differentiation of the governing PDE system (1) with respect to space and time. In the following, we illustrate the Cauchy-Kovalewski procedure symbolically for third order of accuracy. For an efficient implementation up to any order of accuracy in space and time we refer the reader to $[14,13]$. For two more general and fully numerical alternatives to the semi-analytical CauchyKovalewski procedure see $[11,10]$, where local space-time finite element methods are used in order to compute the polynomial $\mathbf{Q}_{i}(x, t)$.

The first time derivative can be directly obtained from (1) as

$\frac{\partial \mathbf{Q}}{\partial t}=-\mathbf{A}(\mathbf{Q}) \frac{\partial \mathbf{Q}}{\partial x}$

The mixed space time derivative is then obtained after a differentiation with respect to space

$\frac{\partial^{2} \mathbf{Q}}{\partial t \partial x}=-\frac{\partial}{\partial x} \mathbf{A}(\mathbf{Q}) \frac{\partial \mathbf{Q}}{\partial x}-\mathbf{A}(\mathbf{Q}) \frac{\partial^{2} \mathbf{Q}}{\partial x^{2}}$,

and the second time derivative of $\mathbf{Q}$ is

$\frac{\partial^{2} \mathbf{Q}}{\partial t^{2}}=-\frac{\partial}{\partial t} \mathbf{A}(\mathbf{Q}) \frac{\partial \mathbf{Q}}{\partial x}-\mathbf{A}(\mathbf{Q}) \frac{\partial^{2} \mathbf{Q}}{\partial t \partial x}$.

The value of $\mathbf{Q}_{i}\left(x_{i}, t^{n}\right)$ and all purely spatial derivatives are obtained from the WENO reconstruction polynomial $\mathbf{w}_{i}\left(x, t^{n}\right)$. 


\subsection{The fully discrete high-order accurate one-step scheme}

Once the WENO reconstruction and the Cauchy-Kovalewski procedure have been performed for each cell, PDE (1) can be integrated over a space-time control volume $\left[x_{i-\frac{1}{2}} ; x_{i+\frac{1}{2}}\right] \times\left[t^{n} ; t^{n+1}\right]$ (see $[5,26]$ for details) and our final high-order accurate one-step scheme can be written as follows:

$\mathbf{Q}_{i}^{n+1}=\mathbf{Q}_{i}^{n}-\frac{1}{\Delta x} \mathbf{A} \mathbf{Q}_{x}-\frac{\Delta t}{\Delta x}\left[\mathbf{D}_{i+\frac{1}{2}}^{-}+\mathbf{D}_{i-\frac{1}{2}}^{+}\right]$,

where

$\mathbf{A Q}_{x}=\int_{t^{n}}^{t^{n+1}} \int_{x_{i-\frac{1}{2}}^{+}}^{x_{i+\frac{1}{2}}^{-}} \mathbf{A}\left(\mathbf{Q}_{i}(x, t)\right) \frac{\partial}{\partial x} \mathbf{Q}_{i}(x, t) d x d t$

and

$\mathbf{D}_{i+\frac{1}{2}}^{ \pm}=\frac{1}{\Delta t} \int_{t^{n}}^{t^{n+1}} \mathbf{A}_{i+\frac{1}{2}}^{ \pm}\left(\mathbf{Q}_{i+\frac{1}{2}}^{+}-\mathbf{Q}_{i+\frac{1}{2}}^{-}\right) d t$

with

$\mathbf{Q}_{i+\frac{1}{2}}^{-}=\mathbf{Q}_{i}\left(x_{i+\frac{1}{2}}, t\right) \quad$ and $\quad \mathbf{Q}_{i+\frac{1}{2}}^{+}=\mathbf{Q}_{i+1}\left(x_{i+\frac{1}{2}}, t\right)$.

All the integrals are approximated using Gaussian quadrature formulae of suitable order of accuracy. Note that the term $\mathbf{A Q}_{x}$, which integrates the smooth part of the non-conservative product within each cell (excluding the jumps at the boundaries), vanishes for a first-order scheme where we have $\frac{\partial}{\partial x} \mathbf{Q}_{i}(x, t)=0$. In the following we briefly summarize the entire high-order one-step algorithm:

(1) Perform the WENO reconstruction described in Section 2.1 in order to obtain the reconstruction polynomials $\mathbf{w}_{i}\left(x, t^{n}\right)$ for each cell.

(2) Compute the spatial derivatives of $\mathbf{w}_{i}\left(x, t^{n}\right)$ and insert them into the Cauchy-Kovalewski procedure in order to get all missing space-time derivatives in the Taylor series (40). This step generates a space-time polynomial $\mathbf{Q}_{i}(x, t)$ for each cell $T_{i}$.

(3) Use the space-time polynomials $\mathbf{Q}_{i}(x, t)$ together with Gaussian quadrature to compute the integrals appearing in the fully discrete scheme (44) and perform the update of the cell averages.

\section{Numerical results}

The PRICE-C scheme presented in this paper is very general and is applicable to any system of hyperbolic equations containing non-conservative products. In this section we assess the performance of the proposed high-order algorithm using the timedependent nonlinear shallow water equations without and with sediment transport as model system.

In the following, numerical results for different test cases are reported. The computations are carried out using a third-order WENO version of the proposed PRICE-C scheme. The Courant number is set to $C F L=0.9$. The matrix (20) has been evaluated using a three-point Gaussian quadrature rule with the following points $s_{j}$ and weights $\omega_{j}$ :

$s_{1}=\frac{1}{2}, \quad s_{2,3}=\frac{1}{2} \pm \frac{\sqrt{15}}{10}, \quad \omega_{1}=\frac{8}{18}, \quad \omega_{2,3}=\frac{5}{18}$.

\subsection{Shallow water equations}

We consider the 1D system of shallow water equations with variable bottom topography. The bottom friction is neglected. The system can be written as: $\frac{\partial H}{\partial t}+\frac{\partial q}{\partial x}=0$

$\frac{\partial q}{\partial t}+\frac{\partial}{\partial x}\left(\frac{q^{2}}{H-b}+\frac{1}{2} g H^{2}-g H b\right)+g H \frac{\partial b}{\partial x}=0$,

where $H=h+b$ is the free surface elevation, $h$ is the water depth, $q=h u$ is the discharge per unit width, $b$ represents the bottom topography and $g$ is the acceleration due to gravity. In order to obtain a well-balanced scheme, we follow the idea developed in $[5,16,17]$. Adding the trivial equation $\partial b / \partial t=0$ in system (49), the problem can be written in the non-conservative form (1), in which the forces due to the variable bottom topography are interpreted as a non-conservative product. The vector $\mathbf{Q}$ and the matrix A assume the following form

$\mathbf{Q}=\left(\begin{array}{c}H \\ q \\ b\end{array}\right), \quad \mathbf{A}=\left(\begin{array}{ccc}0 & 1 & 0 \\ g h-u^{2} & 2 u & u^{2} \\ 0 & 0 & 0\end{array}\right)$,

where $h=H-b$ and $u=q / h$.

We note here that the scheme (44) with matrices (27) and (28) when applied to the shallow water equations produces an artificial motion of the bottom. In fact when the bottom is variable, the component $(3,3)$ of the identity matrix I gives an undesirable diffusion that tends to flatten the bottom also if the water is quiescent. So in the following we use a modified identity matrix $\mathbf{I}_{m}$ that reads:

$\mathbf{I}_{m}=\left[\begin{array}{lll}1 & 0 & 0 \\ 0 & 1 & 0 \\ 0 & 0 & 0\end{array}\right]$

where the undesirable diffusion of the bottom is eliminated.

\subsubsection{Verification of the C-property}

Proof. It is well known that numerical methods for the shallow water system with variable bottom must satisfy the so-called $C$ property as introduced by Bermudez and Vazquez [2]. This means that the term due to the bottom elevation must balance the term due to hydrostatic pressure under quiescent flow conditions over any bottom profile, including discontinuous bottom. For quiescent flow, we have $H=$ const., $u=0$ and therefore

$\Delta \mathbf{Q}=\left(\begin{array}{c}\Delta H \\ \Delta q \\ \Delta b\end{array}\right)=\left(\begin{array}{c}0 \\ 0 \\ \Delta b\end{array}\right), \quad \mathbf{A}_{\Psi}=\left(\begin{array}{ccc}0 & 1 & 0 \\ g \bar{h} & 0 & 0 \\ 0 & 0 & 0\end{array}\right), \quad \mathbf{A}_{\Psi}^{2}=\left(\begin{array}{ccc}g \bar{h} & 0 & 0 \\ 0 & g \bar{h} & 0 \\ 0 & 0 & 0\end{array}\right)$,

with $\bar{h}=\int_{0}^{1} h(s) d s=\int_{0}^{1}\left(h_{L}+s\left(h_{R}-h_{L}\right)\right) d s$. Using the well-balanced identity matrix $\mathbf{I}_{m}$ it follows trivially from Eqs. (26)-(28) and (51) that

$\mathbf{A}_{i-\frac{1}{2}}^{ \pm} \Delta \mathbf{Q}=0$

and therefore the first-order scheme verifies the exact $C$-property. For the higher order scheme (44) we point out that using reconstruction of the free surface elevation $H$ and the bottom topography $b$ leads to a so-called well-balanced reconstruction in the sense of [5], hence also the term $\mathbf{A Q}_{x}=0$.

Numerical verification. The aim of these simulations is now to verify whether also our actual implementation of the proposed PRICE-C scheme in computer code satisfies the exact $C$-property to machine precision. In order to verify this property we perform two different numerical experiments as proposed in [34]. We take 
$b(x)=5 e^{\left(-\frac{2}{5}(x-5)^{2}\right)} \mathrm{m}$

for simulating a smooth bottom and

$b(x)= \begin{cases}4 \mathrm{~m} & \text { if } 4 \mathrm{~m} \leqslant x \leqslant 8 \mathrm{~m}, \\ 0 & \text { otherwise }\end{cases}$

for the discontinuous case. The initial data for both tests are

$H=h+b=10 \mathrm{~m}, \quad q=0$.

To test the ability of the scheme to maintain the initial condition, a simulation is carried out until $t=0.5 \mathrm{~s}$, using a mesh of 200 cells in a $10 \mathrm{~m}$ long domain. We use double precision arithmetics. The errors between numerical and exact solution are given in Table 1, from which we can deduce that the $C$-property is exactly satisfied up to machine precision.

\subsubsection{A small perturbation of a steady state water}

This test was first proposed by LeVeque [22] and aims to assess the capability of the scheme to capture a small pulse propagating over a quiescent state. The bottom topography considered is described by:

$$
b(x)= \begin{cases}0.25 \cos (10 \pi(x-1.5))+1 \mathrm{~m} & \text { if } 1.4 \mathrm{~m} \leqslant x \leqslant 1.6 \mathrm{~m}, \\ 0 & \text { otherwise }\end{cases}
$$

Table 1

Verification of the C-property: water depth and specific discharge norms.

\begin{tabular}{|c|c|c|c|c|}
\hline \multirow[t]{2}{*}{ Test case } & \multicolumn{2}{|l|}{$H$} & \multicolumn{2}{|l|}{$q$} \\
\hline & $L_{1}$ & $L_{\infty}$ & $L_{1}$ & $L_{\infty}$ \\
\hline Test 1 (smooth) & $3.25 e-15$ & $1.12 \mathrm{e}-14$ & $2.42 \mathrm{e}-15$ & $4.56 e-14$ \\
\hline Test 2 (non-smooth) & $4.34 \mathrm{e}-15$ & $1.45 \mathrm{e}-14$ & $7.54 \mathrm{e}-15$ & $3.23 e-14$ \\
\hline
\end{tabular}

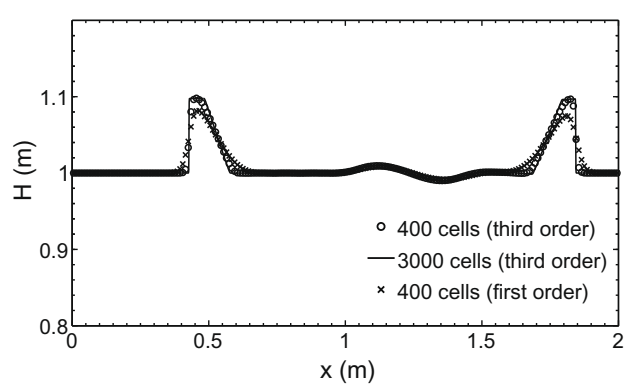

and the initial conditions are:

$q(x, 0)=0$ and $H(x, 0)= \begin{cases}1+\epsilon & \text { if } 1.1 \mathrm{~m} \leqslant x \leqslant 1.2 \mathrm{~m} \\ 1 & \text { otherwise }\end{cases}$

with $\epsilon$ being a small perturbation of the free surface that we choose to be $\epsilon=0.2 \mathrm{~m}$ for the first test and $\epsilon=0.001 \mathrm{~m}$ for the second one. This is a very difficult problem and it is reported in the literature [22] that many numerical schemes fail in computing correctly the propagation of such small perturbations over variable bottom topography. Results for the free surface and the velocity are given in Figs. 2 and 3. The solution obtained using the third-order PRICE-C scheme with 400 cells is compared with a numerical reference solution obtained on a very fine mesh with 3000 cells. The method produces accurate non-oscillatory solutions that are in good agreement with the reference solutions. It is worth noticing that in the case of the small pulse $(\epsilon / h \ll 1)$, theoretically the initial disturbance should split into two waves, propagating to the left and right at the characteristic speed $\sqrt{g h}$. This is correctly reproduced in our numerical simulations.

\subsubsection{Steady flow over a smooth hump}

The aim of such simulations is to analyze the convergence in time towards a steady flow over a smooth bump. To this end we have used three different tests $(a, b, c)$ with exact solution, proposed by the Working group on dam break modeling [18], which are broadly used for testing numerical methods. The bottom topography is the following:

$$
b(x)= \begin{cases}0.2-0.05(x-10)^{2} \mathrm{~m} & \text { if } 8 \mathrm{~m} \leqslant x \leqslant 12 \mathrm{~m}, \\ 0 & \text { otherwise }\end{cases}
$$

while the domain has a length $L=25 \mathrm{~m}$, divided into 200 cells. Steady solutions have been obtained by marching in time to steady state, starting from an initial profile (horizontal free surface profile)

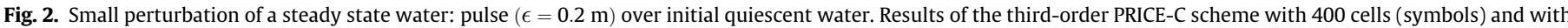
3000 cells (line) at time $t=0.2 \mathrm{~s}$ are shown. Results of the first-order scheme with 400 cells are also shown for comparison.
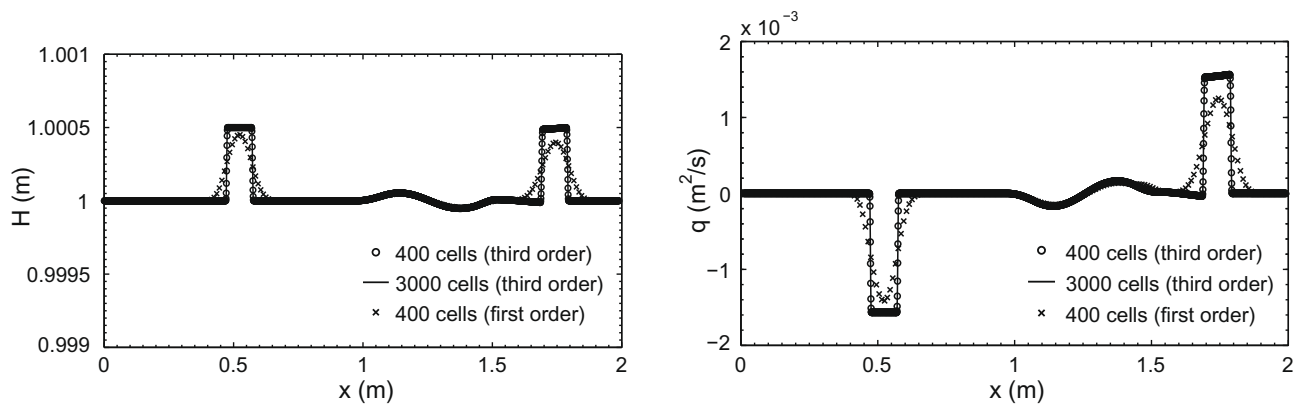

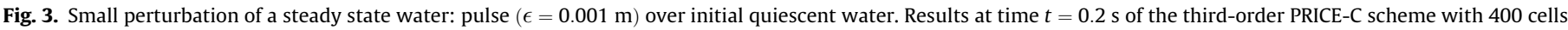
(symbols) and with 3000 cells (line). Results of the first-order scheme with 400 cells are also shown for comparison. 
Table 2

Boundary conditions for the steady flow over a smooth hump.

\begin{tabular}{lll}
\hline Test case & $q(x=0, t)\left(\mathrm{m}^{2} / \mathrm{s}\right)$ & $H(x=L, t)(\mathrm{m})$ \\
\hline (a) & 1.53 & 0.66 \\
(b) & 0.18 & 0.33 \\
(c) & 4.42 & 2.0 \\
\hline
\end{tabular}

that is far away from the steady solution. The initial conditions are taken as

$q(x, 0)=0$ and $H(x, 0)=0.5 \mathrm{~m}$.

Modifying the value of the upstream discharge $q$ or the downstream water surface level $H$ results in different steady configurations and therefore we select different boundary conditions, which are summarized in Table 2. In test case (a) the solution is characterized by a transcritical flow without a shock, for test (b) the solution is characterized by a transcritical flow with a shock, while in test case (c) the solution is given by a completely subcritical flow. The numerical and exact solutions for all test cases are depicted in Fig. 4 at time $t=200 \mathrm{~s}$. The agreement between numerical and exact solution for the free surface elevation $H$ is excellent. No spurious oscillations are produced at the discontinuities and the position of the shock wave is also correct. The small errors that appear in the discharge are also present in other high order schemes documented in the literature, see e.g. [34].
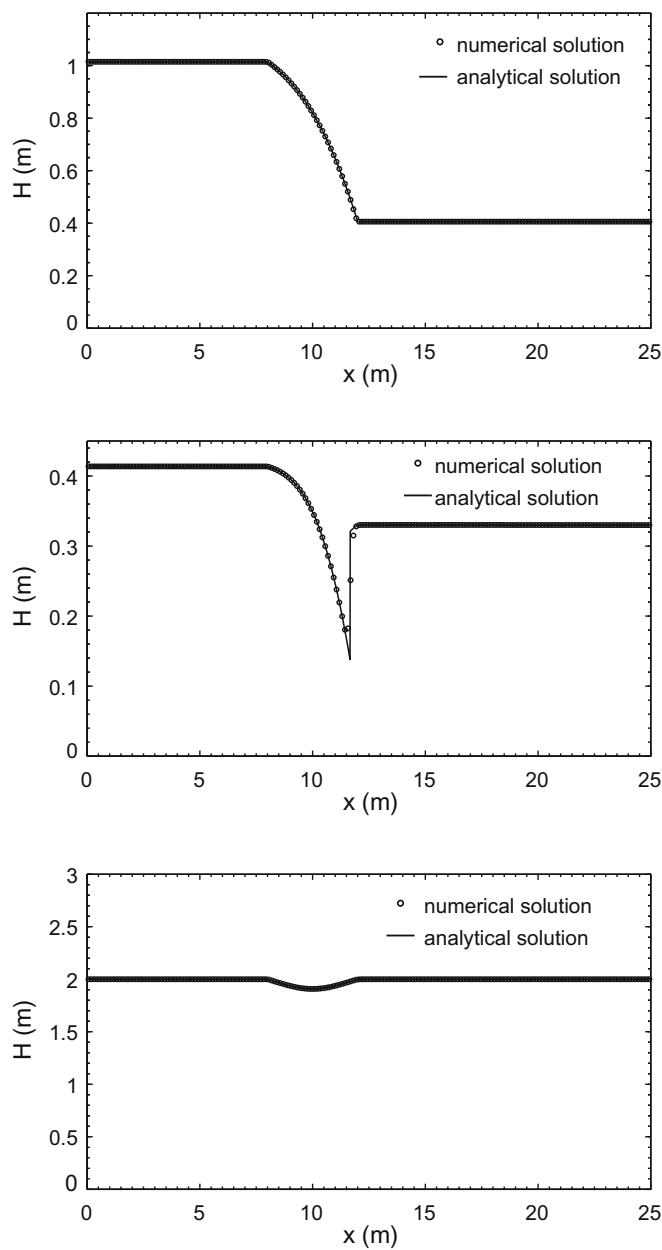

\subsection{Sediment transport}

A system of equations that govern the transport of sediments in gravel bed rivers is obtained coupling the shallow water equations (49) with an equation that describes the bottom evolution, namely the Exner equation. It reads:

$\frac{\partial b}{\partial t}+\frac{\partial q_{s}}{\partial x}=0$

where $b=b(x, t)$ is the movable bed elevation. $q_{s}$ is the bedload sediment transport rate for unit width. Several numerical solutions have been proposed in the literature for this problem $[3,4]$. And for the quantification of $q_{s}$ different relationships are available in the literature. We have used a simple power law for testing the method against exact solutions, namely:

$q_{s}=\frac{A\left(u-u_{c}\right)^{m}}{\left(1-\lambda_{p}\right)}$

where $u$ is the velocity of the water, $u_{c}$ is the critical velocity below which the sediment transport vanishes, $m$ is a positive exponent, while $\lambda_{p}$ is the porosity. Moreover other two empirical formulae available in the literature have been implemented. They are of the type:

$q_{s}=\frac{\sqrt{(s-1) g d_{s}^{3}}}{\left(1-\lambda_{p}\right)} \Phi(\theta)$,
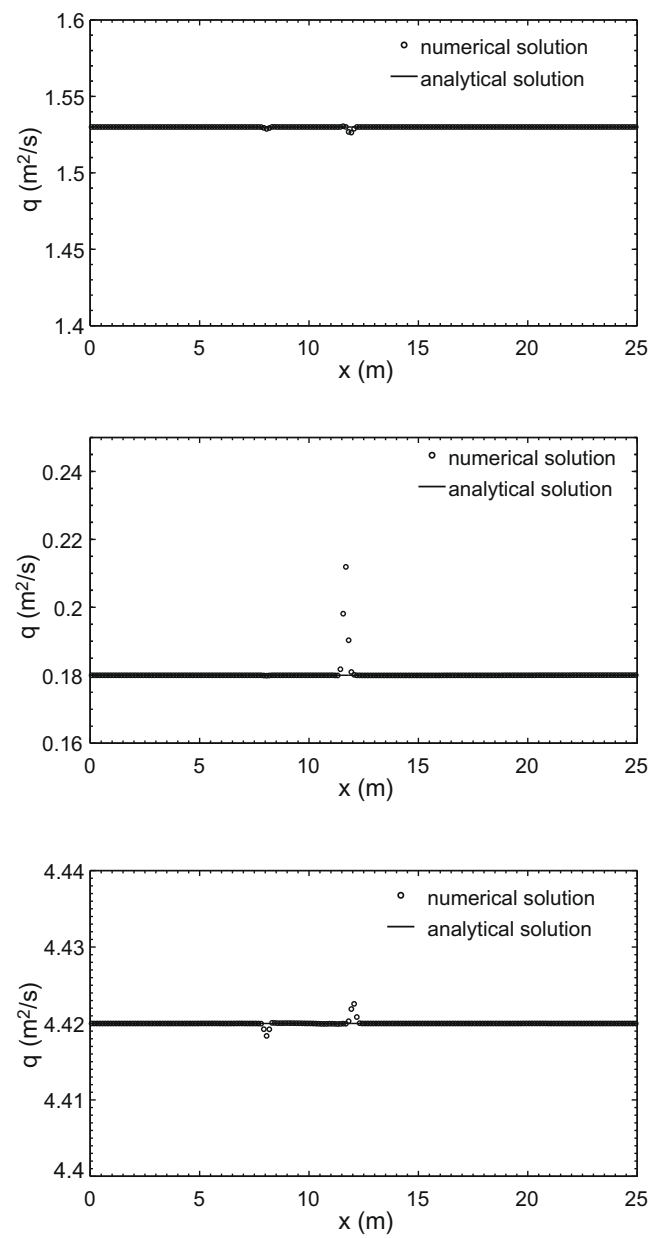

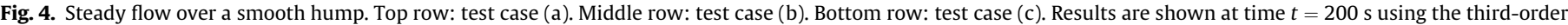
PRICE-C scheme (symbols) as well as the exact solution (line). 
with $s$ being the relative density, and the local Shields stress is given by

$\theta=\frac{S_{f} h}{(s-1) d_{s}}$

where $d_{s}$ is the mean sediment diameter. The friction term $S_{f}$ is calculated using the usual formula of Manning which reads:

$S_{f}=\frac{q^{2} n_{f}^{2}}{h^{10 / 3}}$

$n_{f}$ being the Manning coefficient of roughness. In this paper we make use of the sediment discharge function $\Phi(\theta)$ proposed by Parker [27], which reads

$\Phi=0.00218 \theta^{3 / 2} G(\xi), \quad \xi=\frac{\theta}{\theta_{r}}, \quad \theta_{r}=0.0386$,

with

$G= \begin{cases}5474(1-0.853 / \xi)^{4.5}, & \xi \geqslant 1.59, \\ \exp \left[14.2(\xi-1)-9.28(\xi-1)^{2}\right], & 1 \leqslant \xi \leqslant 1.59, \\ \xi^{14.2}, & \xi<1\end{cases}$

and the one proposed by Meyer-Peter and Müller [25]:

$\Phi= \begin{cases}8(\theta-0.047)^{3 / 2} & \text { if } \theta>0.047 \\ 0 & \text { otherwise. }\end{cases}$

It is worth noticing that the empirical nature of the relationships aiming to quantify the solid discharge $q_{s}$ leads to the availability of a great number of different formulae. As a consequence, each particular choice for the closure relation for $q_{s}$ leads to a different system matrix $\mathbf{A}$ and therefore to a different formulation of the analytical Roe matrix $\mathbf{A}_{\boldsymbol{\Psi}}$. This would result in unsurmountable problems for environmental engineers, whose scope is to try many different available empirical formulations for reproducing field measurements or laboratory experiments. The main advantage of the proposed PRICE-C method with the fully numerical computation of the centred Roe-type matrix $\mathbf{A}_{\Psi}^{\mathbf{M}}$ via Gaussian quadrature along the path is that it completely avoids the need for an explicit computation of the Roe averages, at the same time being accurate up to the prescribed order for any choice of the solid transport formula.

The system of governing equations describing the coupled evolution of the fluid and the bed can be written in the form (1), see [28], with the vector $\mathbf{Q}$ and matrix $\mathbf{A}$ being respectively:

$\mathbf{Q}=\left(\begin{array}{c}H \\ q \\ b\end{array}\right), \quad \mathbf{A}=\left(\begin{array}{ccc}\frac{\partial q_{s}}{\partial H} & 1+\frac{\partial q_{s}}{\partial q} & \frac{\partial q_{s}}{\partial b} \\ g h-u^{2} & 2 u & u^{2} \\ \frac{\partial q_{s}}{\partial H} & \frac{\partial q_{s}}{\partial q} & \frac{\partial q_{s}}{\partial b}\end{array}\right)$.

In the following we show the results provided by the proposed PRICE-C scheme for three different test cases.

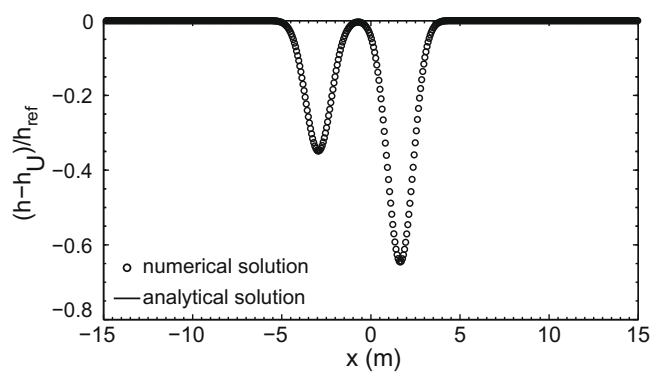

3.2.1. Propagation of a small sediment hump near critical conditions

A test aiming to reproduce bed movement near critical conditions is carried out. Under these conditions, the coupling between the shallow water equations and the Exner equation within the time step is mandatory. In this range, in fact, each of the wave propagation speeds can no longer be identified solely with a surface wave or solely with a bed wave, and a full coupling of the equations is necessary to correctly solve the propagation of bed disturbances. The initial Froude number is taken as $\mathrm{Fr}_{U}=0.979$, where $U$ indicates the uniform unperturbed state. The initial bed topography is described by:

$b(x, 0)=b_{\max } e^{-x^{2}} \mathrm{~m}$ with $-15 \mathrm{~m} \leqslant x \leqslant 15 \mathrm{~m}$,

where $b_{\max }=10^{-5} \mathrm{~m}$ is the amplitude of the initial bed perturbation. The initial condition is obtained running the code with a fixed bed configuration. The upstream discharge is fixed according to the Froude number, while downstream a fixed water depth equal to $1 \mathrm{~m}$ is imposed. The domain length $L=25 \mathrm{~m}$ is divided into spatial steps of $0.05 \mathrm{~m}$, leading to 500 cells. The numerical results are compared with an exact solution, obtained by Lyn and Altinakar [24] linearizing the governing system of equations using a small parameter $\psi_{U}$ defined as follows:

$\psi_{U}=\frac{1}{\left(1-\lambda_{p}\right) h_{U}} \frac{\partial q_{s}}{\partial u}$.

The adopted sediment transport formula is of the form (62) in which $A=3.4 \times 10^{-4}$ and $m=2.65$, while $u_{c}$ is determined solving Eq. (71) setting $\psi_{U}=2.5 \times 10^{-3}$. In Fig. 5 the comparison between numerical and analytical solution is given for $t=20 \mathrm{~s}$ for both the bottom and the water surface. The quantities are plotted in dimensionless form, with the scaling parameters being:

$h_{\mathrm{ref}}=\frac{b_{\mathrm{max}}}{\left(1-F r_{U}^{2}\right)}, \quad b_{\mathrm{ref}}=b_{\max }$.

We note two waves generated on the bottom: the scour wave propagating upstream and a depositional wave propagating downstream, while in correspondence of the bottom disturbances two negative waves are generated in the water depth $h$. The numerical solution obtained with the third-order PRICE-C scheme is in excellent agreement with the analytical solution presented in [24].

\subsubsection{Propagation of a sediment bore}

In this test case we apply different transport formulae comparing numerical results with those obtained experimentally in [1]. The experiment consists in a steep-sloped, rectangular channel of finite length. The bed profile is in quasi-equilibrium and a constant sediment supply is fed upstream. At reference time $t=0$, this equilibrium situation is perturbed by the rapid raise of a submerged weir at the downstream end of the flume, imposing a subcritical condition. The water and sediment discharges at the upstream section are kept constant. This hydraulic configuration gives rise to a

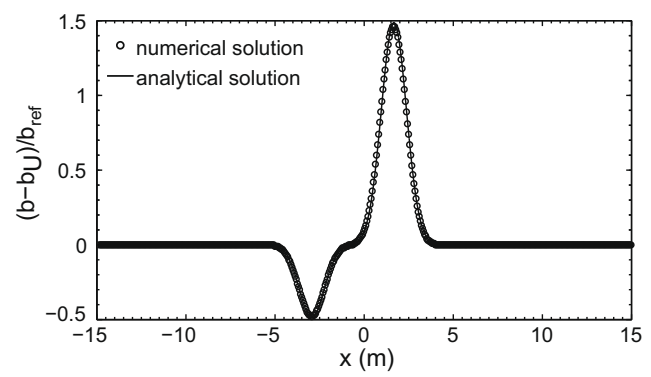

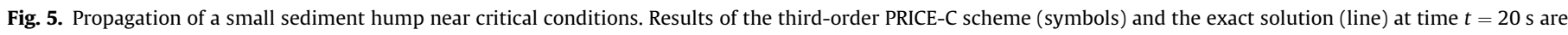
shown. 


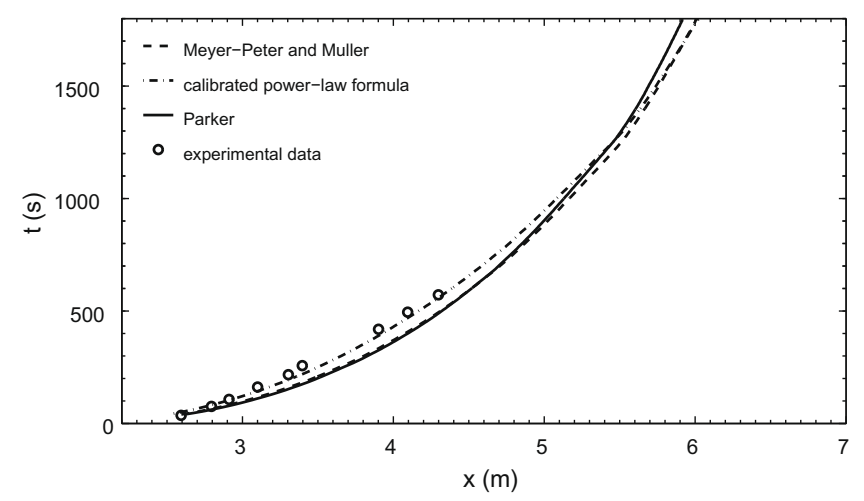

Fig. 6. Comparison of front position for the third-order PRICE-C scheme using three different formulae for the quantification of the solid discharge.

hydraulic jump and a sediment bore. The aggradational shock front caused by the presence of transcritical flow represents a demanding test case in which numerical schemes may fail in predicting both intensity and propagation velocity of the front itself. The flume is $6.9 \mathrm{~m}$ long, $0.50 \mathrm{~m}$ wide and the slope is equal to $3.02 \%$. The sediment and water discharges are $q_{s}=0.136 \mathrm{l} / \mathrm{s}$ and $Q=12 \mathrm{l} / \mathrm{s}$, respectively. The induced water level at the downstream end is $H=20.93 \mathrm{~cm}$; uniform coarse sand with a mean diameter of $1.65 \mathrm{~mm}$ and with a porosity of 0.42 is considered. Finally, Manning coefficient is $n_{f}=0.0165 \mathrm{~m}^{3} \mathrm{~s}$. Numerical treatment of the friction term is made by using the approach of Gosse [16,17], which leads to the following system of governing equations:

$\mathbf{Q}=\left(\begin{array}{c}H \\ q \\ b \\ x\end{array}\right), \quad \mathbf{A}=\left(\begin{array}{cccc}\frac{\partial q_{s}}{\partial H} & 1+\frac{\partial q_{s}}{\partial q} & \frac{\partial q_{s}}{\partial b} & 0 \\ g h-u^{2} & 2 u & u^{2} & g h S_{f} \\ \frac{\partial q_{s}}{\partial H} & \frac{\partial q_{s}}{\partial q} & \frac{\partial q_{s}}{\partial b} & 0 \\ 0 & 0 & 0 & 0\end{array}\right)$.

Numerical simulations are conducted using the sediment transport formulae (66) and (68) and a formula of the type (62) calibrated with parameters $A=0.00024, m=3$ and $u_{c}=0.3 \mathrm{~m} / \mathrm{s}$. In Fig. 6 the position of the sediment bore obtained with all three different sediment discharge formulae is plotted as a function of time and is compared with the experimental data. Shock position at time $t^{n+1}$ is defined as the $x$ coordinate of the barycentre of the first cell (starting from the right boundary) that satisfies:

$b_{i}^{n+1}-b_{i}^{n}>\tau$

$\tau$ is a given tolerance fixed to $0.02 \mathrm{~m}$ in all the computations and for all the different sediment formulae. The celerity of the front is given by the inverse of the slope of the above curves. As it is seen the propagation celerity depends on the transport formula used. This means that thanks to its generality and simplicity, the numerical tool that we propose can be very useful for practitioners when they want to reproduce real data because they can test many different transport formulae without having to adapt the numerical method to each specific closure relation for $q_{s}$.

\subsection{Numerical convergence study}

In the previous sections we have shown that the proposed numerical method well reproduces unsteady solutions and the results are essentially non-oscillatory. Here we compute the order of accuracy of the scheme to verify that the expected theoretical order is achieved. We solve the inviscid shallow water equations coupled with a bottom evolution equation, written with respect to the variables $h, q$ and $b$, that reads:

$$
\left\{\begin{array}{l}
\partial_{t} h+\partial_{x} q=0, \\
\partial_{t} q+\partial_{x}\left(q u+\frac{1}{2} g h^{2}\right)=-g h \partial_{x} b, \\
\partial_{t} b+\partial_{x} q_{s}=0 .
\end{array}\right.
$$

In order to validate the order of accuracy an exact solution is constructed by prescribing three functions for $h(x, t), q(x, t)$ and $b(x, t)$ which satisfy exactly (75). They read

$$
\begin{aligned}
h(x, t) & =h_{0}+c_{0} \sin (k x-\omega t), \quad q(x, t) \\
& =\frac{\omega}{k} h_{0}+c_{0} \frac{\omega}{k} \sin (k-\omega t), \quad b(x, t) \\
& =-h(x, t), \quad q_{s}(x, t)=-q(x, t), \quad k=\frac{2 \pi}{L_{w}}, \quad \omega=\frac{2 \pi}{T_{p}} .
\end{aligned}
$$

We underscore that the relation $q_{s}=-q$ is not physically based, but it allows us to find an exact solution of system (75). Table 3 shows the errors quantified through the standard norms $L_{1}, L_{\infty}$ and relative convergence rates for variables $h$ and $q$ at the time $t=100 \mathrm{~s}$ with $c_{0}=0.01 \mathrm{~m}, h_{0}=5 \mathrm{~m}, T_{p}=10 \mathrm{~s}, L_{w}=25 \mathrm{~m}$. We can see that the third order of accuracy is achieved with each norm.

\section{Conclusions}

We have presented a first-order monotone centred scheme, called PRICE-C, which is based on the centred FORCE scheme for conservation laws [31,32]. It can be seen as an extension of the PRICE-T method proposed in [29] using the insights gained by the path-conservative methods developed recently in $[5,26]$. We have extended this first-order method to third order of accuracy in space and time via the ADER approach using a WENO reconstruction technique. Extensive numerical experiments suggest that the scheme is very general, though efficient and simple. It yields very satisfactory results compared to exact and experimental reference solutions. The first attractive feature of the presented method is the simplicity due to an approximate computation of the Roe matrix via Gaussian quadrature rules of suitable order of accuracy. In practice, we found that for shallow-water-type PDE systems, three Gaussian points seem to be enough to ensure conservation. This avoids the need for an analytical Roe matrix. A second important aspect concerns the future extension of the method to multi-

\begin{tabular}{|c|c|c|c|c|c|c|c|c|}
\hline \multirow[t]{2}{*}{$N$} & \multicolumn{4}{|l|}{ Variable $h$} & \multicolumn{4}{|l|}{ Variable $q$} \\
\hline & $L_{1}$ & $\mathcal{O}\left(L_{1}\right)$ & $L_{\infty}$ & $\mathcal{O}\left(L_{\infty}\right)$ & $L_{1}$ & $\mathcal{O}\left(L_{1}\right)$ & $L_{\infty}$ & $\mathcal{O}\left(L_{\infty}\right)$ \\
\hline 20 & $5.54 \mathrm{E}-03$ & & $8.57 \mathrm{E}-03$ & & $1.39 \mathrm{E}-02$ & & $2.14 \mathrm{E}-02$ & \\
\hline 40 & $1.71 \mathrm{E}-03$ & 1.70 & $2.70 \mathrm{E}-03$ & 1.67 & $4.27 \mathrm{E}-03$ & 1.70 & $6.75 \mathrm{E}-03$ & 1.67 \\
\hline 80 & $2.45 \mathrm{E}-04$ & 2.80 & $3.86 \mathrm{E}-04$ & 2.81 & $6.13 \mathrm{E}-04$ & 2.80 & $9.64 \mathrm{E}-04$ & 2.81 \\
\hline 160 & $3.05 E-05$ & 3.01 & $4.79 \mathrm{E}-05$ & 3.01 & $7.62 \mathrm{E}-05$ & 3.01 & $1.20 \mathrm{E}-04$ & 3.01 \\
\hline 320 & $3.62 \mathrm{E}-06$ & 3.08 & $5.68 \mathrm{E}-06$ & 3.08 & $9.04 \mathrm{E}-06$ & 3.08 & $1.42 \mathrm{E}-05$ & 3.08 \\
\hline 640 & $4.01 \mathrm{E}-07$ & 3.17 & $6.30 \mathrm{E}-07$ & 3.17 & $1.00 \mathrm{E}-06$ & 3.17 & $1.57 \mathrm{E}-06$ & 3.17 \\
\hline
\end{tabular}
ple space dimensions: this can be achieved since the matrices $\mathbf{A}_{i+\frac{1}{2}}^{ \pm}$

Table 3

Convergence rates study for the sediment transport problem with source terms for the third-order PRICE-C method $\left(c_{0}=0.01 \mathrm{~m}, h_{0}=5 \mathrm{~m}, T_{p}=10 \mathrm{~s}, L_{w}=25 \mathrm{~m}\right)$. 
of the PRICE-C method have been modified in such a way as to become two-point functions of the two adjacent states, in contrast to the original PRICE-T method or the PRICE-R scheme shown in this paper, where these matrices were dependent on three states.

The high-order centred schemes presented here are very general and can be applied to any hyperbolic system in non-conservative form that may exhibit at the same time smooth and discontinuous solutions. The advantage of the presented centred scheme over upwind-based methods is its simplicity and efficiency, and will be fully realized for hyperbolic systems in which the provision of upwind information is very costly or is not available.

\section{Acknowledgement}

The first author thanks Cariverona for financial support under the project MODITE.

\section{References}

[1] Bellal M, Spinewine C, Zech Y. Morphological evolution of steep-sloped river beds in the presence of a hydraulic jump. In: Experimental study, paper presented at XXX IAHR congress, 2003. p. 133-40.

[2] Bermudez A, Vazquez E. Upwind methods for hyperbolic conservation-laws with source terms. Comput Fluids 1994;23(8):1049-71.

[3] Caleffi V, Valiani A. High-order balanced cweno scheme for movable bed shallow water equations. Adv Water Resour 2007;30:730-41.

[4] Castro M, Ferndandez-Nieto ED, Ferreiro A. Sediment transport models in shallow water equations and numerical approach by high order finite volume methods. Comput Fluids 2008;37(3):299-316.

[5] Castro M, Gallardo J, Parés C. High-order finite volume schemes based on reconstruction of states for solving hyperbolic systems with nonconservative products applications to shallow-water systems. Math Comput 2006;75: 1103-34.

[6] Castro M, LeFloch P, Muñoz ML, Parés C. Why many theories of shock waves are necessary: convergence error in formally path-consistent schemes. J Comput Phys 2008;227:8107-29.

[7] Castro M, Pardo A, Parés C, Toro E. Coefficient-splitting numerical schemes for nonconservative hyperbolic systems and high order extensions, submitted for publication.

[8] Chen G, Toro E. Centered difference schemes for nonlinear hyperbolic equations. J Hyperbolic Diff Eq 2004;1(3):531-66.

[9] DalMaso G, LeFloch P, Murat F. Definition and weak stability of nonconservative products. J Math Pure Appl 1995;74(6):483-548.

[10] Dumbser M, Balsara D, Toro E, Munz C. A unified framework for the construction of one-step finite-volume and discontinuous Galerkin schemes. J Comput Phys 2008;227:8209-53.

[11] Dumbser M, Enaux C, Toro E. Finite volume schemes of very high order of accuracy for stiff hyperbolic balance laws. J Comput Phys 2008;227: 3971-4001.
[12] Dumbser M, Käser M. Arbitrary high order non-oscillatory finite volume schemes on unstructured meshes for linear hyperbolic systems. J Comput Phys 2007;221:693-723.

[13] Dumbser M, Käser M, Titarev V, Toro E. Quadrature-free non-oscillatory finite volume schemes on unstructured meshes for nonlinear hyperbolic systems. J Comput Phys 2007;226:204-43.

[14] Dumbser M, Munz C. Building blocks for arbitrary high order discontinuous Galerkin schemes. J Sci Comput 2006;27:215-30.

[15] Glimm J. Solution in the large for nonlinear hyperbolic systems of equations. Commun Pure Appl Math 1965;18:697-715.

[16] Gosse L. A well-balanced flux-vector splitting scheme designed for hyperbolic systems of conservation laws with source terms. Comput Math Appl 2000;39(9-10):135-59.

[17] Gosse L. A well-balanced scheme using non-conservative products designed for hyperbolic systems of conservation laws with source terms. Math Model Methods Appl Sci 2001;11(2):339-65.

[18] Goutal N, Maurel F. In: Proceedings of the second workshop on dambreakwave simulation. Technical Report HE-43/97/016/A, Electricité de France, Département Laboratoire National d'Hydraulique, Groupe Hydraulique Fluviale, 1997.

[19] Harten A, Engquist B, Osher S, Chakravarthy S. Uniformly high order essentially non-oscillatory schemes III. J Comput Phys 1987;71:231-303.

[20] Hou T, LeFloch P. Why non-conservative schemes converge to the wrong solutions: error analysis. Math Comput 1994;62:497-530.

[21] Jiang G, Shu C. Efficient implementation of weighted ENO schemes. J Comput Phys 1996;126:202-28.

[22] LeVeque R. Balancing source terms and flux gradients in high-resolution Godunov methods: the quasi-steady wave-propagation algorithm. J Comput Phys 1998;146(1):346-65.

[23] Liu X, Osher S, Chan T. Weighted essentially non-oscillatory schemes. J Comput Phys 1994;115:200-12.

[24] Lyn D, Altinakar M. St. Venant Exner equations for near-critical transcritical flows. J Hydraul Eng, ASCE 2002;128(6):579-87.

[25] Meyer-Peter E, Müller R, Formulas for bed-load transport. In: Report on 2nd meeting on international association on hydraulic structures research, Stockholm, 1948. p. 39-64.

[26] Parés C. Numerical methods for nonconservative hyperbolic systems: a theoretical framework. SIAM J Numer Anal 2006;44:300-21.

[27] Parker G. Surface-based bedload transport relation for gravel rivers. J Hydraul Res 1990;28(4):417-36.

[28] Siviglia A, Nobile G, Colombini M. Quasi-conservative formulation of the one dimensional Saint Venant-Exner model. J Hydraul Eng 2008;134(10):1521-6.

[29] Toro E, Siviglia A. PRICE: primitive centred schemes for hyperbolic system of equations. Int J Numer Methods Fluids 2003;42:1263-91.

[30] Toro E, Titarev V. Solution of the generalized Riemann problem for advectionreaction equations. Proc Roy Soc Lond Ser A - Math Phys Eng Sci 2002;458(2018):271-81.

[31] Toro EF. On Glimm-Related Schemes for Conservation Laws. Technical Report MMU-9602, Department of Mathematics and Physics, Manchester Metropolitan University, UK, 1996.

[32] Toro EF, Billett SJ. Centred TVD schemes for hyperbolic conservation laws. IMA J Numer Anal 2000;20:47-79.

[33] Toumi I. A weak formulation of Roe's approximate riemann solver. J Comput Phys 1992;102(2):360-73.

[34] Xing Y, Shu C. High-order well-balanced finite difference WENO schemes for a class of hyperbolic systems with source terms. J Sci Comput 2006;27(13):477-94. 